\title{
Conceptualizing Interactivity on Social Media and Exploring the Effects of Interactivity on Consumers' Engagement with Online Social-Interactions
}

\author{
Fei Qiao \\ School of Journalism and Communication, Guangdong University of Foreign Studies, CHINA \\ (iD) 0000-0002-5322-4847 \\ jennifer.qf@gmail.com
}

ARTICLE INFO

Received: 20 February 2019

Accepted: 7 May 2019

Published: 14 May 2019

DOI: https://doi.org/10.29333/ojcmt/5781

ABSTRACT

With the rapid acceptance of using social media to achieve interactive engagement come varied opportunities for businesses to communicate with their customers and clients. Through an analysis of McDonald's "Our Food, Your Questions" campaign on social media platforms, our aim for the present study is two-fold. First, we conceptualize interactivity on the content-community level of social media on a continuum from low- to middle- to high-order, and we distinguish between medium-based interactivity, conversation-based interactivity, and process-based interactivity. Second, we examine the effects of these interactive strategies on users. The results show that conversation-based interactivity generates greater participation as well as emotional and social-engagement than does medium-based interactivity. Additionally, process-based interactivity produces more positive, emotional-social engagement than do either conversation-based interactivity or medium-based interactivity. We conclude by discussing the theoretical and practical implications for advancing our knowledge of interactivity on social media and marketing practices.

Keywords: interactivity conceptualization, medium-based interactivity, process-based interactivity, conservation-based interactivity, social-interactivity, content-community level of social media

\section{INTRODUCTION}

As social media platforms are offering unprecedented convenience for businesses to reach their consumers, the communication between corporate entities and their consumers has undergone drastic changes over the past ten years. According to Facebook, until the present, nearly 50 million businesses use Facebook Pages to connect with their stakeholders (Hainla, 2018). Furthermore, around 33\% of customers prefer to talk with companies on social media over telephone or mail, and $57 \%$ of consumers report that they are more likely to think highly of a particular business if they see more positive comments on social media (Aguilhar, 2018). All of this is part of a broad trend toward incorporating social media platforms into businesses' interactive strategies to attract new consumers, to retain current customers, and, more importantly, to engage their participation.

In 2014, McDonald's launched a continuous global campaign named "Our Food, Your Questions" to improve its transparency and to enhance engagement with their customers 
(Starkman, 2014). They invite people around the world to post their questions pertaining to McDonald's food quality on social media platforms, including on their official Facebook page, their official Twitter account, and their official YouTube Page. McDonald's assigns personnel to directly reply to those questions. At present, this Q\&A section on McDonald's official social media platforms is still accessible, and consumers are still able to ask questions.

We observed three types of relational maintenance strategies that McDonald's has used on social media. First, the corporation provides some people who ask questions only with hyperlinks, directing them to find answers via clicking on appropriate links. Second, they sometimes start brief conversations with people, forming a virtual communicative flow. Third, they offer both hyperlinks and brief conversations to answer some people's questions. These three types of relational maintenance strategies represent different dimensions of interactivity on social media. Yet, except for Kelleher (2009), whose works we have reviewed below, few previous authors have focused exclusively on exploring interactivity on social media or provided more accurate and multi-dimensional definitions of interactivity specifically for social media platforms. With the rapid growth of social media comes the need and opportunity to offer a clearer and more informative definition of interactivity. Therefore, we aim to contribute to this opportunity, offering the following:

a. Conceptualizing interactivity on social media

b. Examining the effects of interactivity on consumers' engagement with online social interactions.

\section{LITERATURE REVIEW}

\section{Social Media}

According to Kaplan and Haenlein (2009): "Social Media is a group of Internet-based applications that build on the ideological and technological foundations of Web 2.0, and that allow the creation and exchange of User-Generated Content" (p. 61).

The formal definition of social media involves two related concepts: Web 2.0 and UserGenerated Content (Kaplan \& Haenlein, 2009). Web 2.0 is a term first used in 2004 to describe a platform whereby all users can change content and applications in an active and cooperative way instead of only particular users (Kaplan \& Haenlein, 2009). UserGenerated Content (UGC) is a term people widely used in 2005 to describe the different kinds of media content available to the public and created by all users collaboratively (Kaplan \& Haenlein, 2009). The formal definition of social media incorporates both terms, Web 2.0 and UGC.

There are several ways to categorize social media. One systematic way to categorize social media is based on the social-presence theory and on the media-richness theory (Kaplan \& Haenlein, 2009). In the social presence theory, different media have different degrees of achievement in the acoustic, visual, and physical contact between two communication partners (Kaplan \& Haenlein, 2009; Short, Williams, \& Christie, 1976). The more acoustic, visual, and physical contact the media achieves, the higher its level of social presence. In the media richness theory, different media have different degrees of information transmission in a given time interval (Daft \& Lengel, 1986; Kaplan \& Haenlein, 2009).

Within the classification of the social presence theory and the media richness theory, social media fall into one of three levels (Kaplan \& Haenlein, 2009). The lowest level includes collaborative projects and blogs, like Wikipedia, because such applications are 
text-based and only allow for a relatively simple information exchange. The second level is the community-content level, which includes applications such as Facebook, because the applications in this level allow people to share pictures, videos, and other forms of media. The highest level includes virtual games and social worlds because the applications in this level try to simulate real-life, face-to-face communication in a virtual world. Social media scholars assume that the greater the information transmitted in a period of time, the higher the level of media richness (Kaplan \& Haenlein, 2009).

Interactivity

Different scholars use different conceptualized and operational definitions for interactivity. McMillan (2002) has grouped these approaches into three different categories and labels them "structure,"“user," and "process" (Tremayne, 2008). Their definition of "structure interactivity" emphasizes the structural characteristics of new media that facilitate an interactive exchange. They measure interactivity by collecting information from websites' structural features like users' browsing cookies (Aikat, 2000). Their definition of "user interactivity" involves examining how interactive computermediated communication (CMC) influences users (Tremayne, 2008). Previous scholars have been more interested in investigating the cognitive effects of CMC on users (Salomom, 1997; Walther, 1994). Their definition of "process interactivity" involves looking at the communication process of interactivity. Rafaeli and Sudweeks (1997) even argued that "interactivity is not a characteristic of the medium. It is a process-related construct about communication" (p. 5). Rafaeli and Sudweeks' (1997) claimed that one can only find interactivity in the exchange between parties. Liu and Shrum (2002) upheld Rafaeli and Sudweeks' conceptualization of interactivity and proposed a more formal definition of interactivity. According to Liu and Shrum (2002), interactivity is "the degree to which two or more communication parties can act on each other, on the communication medium, and on the messages and the degree to which such influences are synchronized"(p. 54). While scholars have widely accepted Rafaeli and Sudweeks' "process interactivity," few have operationalized the interactivity he proposed or measured its effects (Tremayne, 2005).

In terms of examining interactivity on social media, Kelleher (2009) applied Sundar, Kalyanaraman, and Brown's (2003) conceptualization of interactivity to examine people's perceptions toward organizations after commenting on the organizations' blogs, which belong to the lowest level of social media, as reviewed in the previous section. Sundar et al. (2003) stated that "a functional view of interactivity is basically an interface's capacity for conducting a dialogue or information exchange between users and the interface" ( $p$. 33), which suggests that the design of a webpage can facilitate web-based communication. These authors argued that the functions of the medium on the interface, such as event calendars, survey polls, and downloadable information, are of use to form dialogic loops, but they fail to push the dialogic potential, which means these companies have the potential to begin an actual dialogue with their customers.

To advance the conceptualization of interactivity, Sundar et al. (2003) added another dimension-contingency interactivity, which they defined as "a process involving users, media, and messages in which communication roles need to be interchangeable for full interactivity to occur" (p. 34-35). Sundar et al.'s (2003) contingency interactivity is also consistent with Rafaeli and Sudweeks' (1997) process interactivity. Kelleher (2009) summarized saying that it is "more that one person's response to another depends on the content of the previous exchanges between the two, and the more intertwined and cumulative, and the more fully interactive the process is said to be" (p. 174). Contingency 
interactivity simulates verbal interactivity, forming a virtual face-to-face communication flow between two parties. Kelleher (2009) found that the level of contingency interactivity that users experience positively correlates with relational outcomes, such as trust and satisfaction, etc., yet, Kelleher (2009) did not conduct an analysis on the effects of functional interactivity on users. Beyond that, Kelleher's (2009) only focused his research on the lowest level of social media, not expanding it to the higher level of social media platforms, like Facebook or Twitter, the more permeable and commonly-adopted application in today's media era. In the current study, we attempt to conceptualize the types of interactivity that are unique to a higher level of social media and of better use.

\section{Conceptualization of Interactivity on The Content-Community Level of Social} Media

As reviewed above, the second level of social media, which includes Facebook and Twitter, is content communities, which allows businesses to connect with users directly via commenting and replying. Even though users can also comment on some organizations' blogs, they don't typically receive a response from a staff member (Kelleher, 2009). Therefore, Sundar et al.'s (2003) functional interactivity has one main constraint that one cannot easily apply to the content-community level of social media. According to Sundar et al. (2003), interface capacity refers to the features incorporated in the design of the website, and users rely on those features to obtain information without assistance from the businesses, the other party involved in the interactive process. In other words, Sundar et al.'s (2003) functional interactivity is not a dynamic process. For example, users need to locate information on their own through event calendars or downloadable information. Yet, in the content-community level of social media, the main features allow users to socialize, to talk, and to share (Mersey, Malthouse, \& Calder, 2010). Businesses utilize these characteristics to connect with users, and users expect to obtain information shared directly by businesses on this type of social media. Therefore, Sundar et al.'s (2003) functional interactivity cannot fully express or include this function of the contentcommunity level of social media. Furthermore, even though Sundar et al. (2003) introduced contingency interactivity, which focuses on virtual, verbal communication between two parties, contingency interactivity might not be inclusive of this type of social media. In certain situations, brief conversations might not satisfy users; they might also want links, photos, or videos shared by the business to feel fully involved in the whole interactive process.

As we mentioned in the introduction, McDonald's relational maintenance strategies include three main types: a) providing users with hyperlinks, b) starting brief conversations with users, and c) using both hyperlinks and brief conversations. In the current study, we integrate previous studies with the observation of McDonald's relational maintenance strategies, and we propose the following conceptualization of interactivity based on shared content through the content-community level of social media.

a. Medium-based interactivity. This is a low-order level of interactivity that consists of the sharing of various mediums such as hyperlinks, photos, videos, emojis, etc. to help consumers to pinpoint information they've inquired about.

b. Conversation-based interactivity. This is middle-order level of interactivity involving businesses starting a brief conversation with consumers. The back and forth between the two parties forms a virtual face-to-face talk. 
c. Process-based interactivity. This is a high-order level of interactivity that includes both medium-based and conversation-based interactivity. As in a real and serious communication, businesses not only "talk" with consumers but also show "proof" to them. Brief conversations and medium-level sharing together generate a genuine processrelated feeling to engage consumers.

To sum up, in this study, we view the ability to share content in a dynamic manner as a key characteristic of the content-community level of social media, which distinguishes it from other levels of social media. We theorize a continuum of interactivity, from low to middle to high orders, that characterizes deeper interactivity by the sharing of more media types and the starting of more conversations with consumers.

\section{Social-interactive Engagement}

Social-interactive engagement represents interactive features that are available on social media at the content community level (Ksiazek, Peer, \& Lessard, 2016). Mersey, Malthouse, and Calder (2010) stated that social-interactive engagement involves two aspects: participation and socializing.

One can measure participation through quantifiable indicators on social media sites, such as online commenting (Mersey et al., 2010), and the numbers of "likes" and "upvotes." Online commenting is the public's participation and socializing, and its presence on sites suggests that the public is willing or has the behavioral intention to be involved in the interactive communication and to contribute to their own online community. In other words, the more online comments that stakeholders produce, the more engaged they are (Wang, Qiao, \& Peng, 2015). In addition, "likes" and "upvotes" also serve an important indicator for stakeholders' online engagement (Gerlitz \& Helmond, 2013). On Facebook, "like" refers to users' agreement with or approval of the implicated comments (Facebook, 2017). The more users who "like" the comments, the more users are engaged (Gerlitz \& Helmond, 2013).

Socializing involves the quality of users' emotions, which one can measure through analyzing comments that users post (Wang et al., 2015), which suggests that interactivity affects stakeholders from the perspective of emotion.

\section{Research Questions}

Based on previous literature, we propose the following research questions.

RQ1: What are the different effects of three types of interactivity on consumers' participative social-interactive engagement?

RQ2: What are the different effects of three types of interactivity on consumers' emotional social-interactive engagement?

\section{METHODS}

\section{Selection of Social Media Sites}

In 2014, McDonald's launched its campaign on two social media sites, Twitter and Facebook, and they started to reply to the public's questions on these two sites. The design of Facebook allows all companies to choose if everyone can view comments without taking any further steps. Similarly, users can retweet posts on Twitter, but all comments are not easily accessible. Thus, to better investigate the research questions, we selected Facebook.

\section{Sampling}

McDonald's has been constantly encouraging the public to ask questions about their products. At present, Facebook customers have asked about a total of five McDonald's products including their beef patties, McRibs, Chicken McNuggets, fries, and eggs. For 
each product, we randomly selected 200 comments, leading to our analyzing a total of 1,000 comments on Facebook.

Unit of Analysis

A comment by one user is one unit of analysis. We used $15 \%$ of the posts to calculate intercoder reliability, and two trained coders coded all sampled comments. We calculated intercoder reliability based on Cohen's Kappa, ranging from .81 to 1.0. We report the intercoder reliability after each measured variable. The codebook is in the Appendix.

\section{Operational Definitions/Measured Variables}

Medium-based interactivity. In this study, we used the operational definition of medium-based interactivity to describe McDonald's shared hyperlinks to answer users' questions on Facebook."Hyperlinks" refers to "a word, phrase, or image that you can click on to jump to a new document or a new section within the current documents."(TechTerms.com, 2014, p. 1). On Facebook, hyperlinks allow users to click and jump from one page to another page. When McDonald's used hyperlinks to answer a question, then we assigned the number " 1 ."When they did not use a hyperlink to answer the question, then we assigned the number "0."The intercoder reliability is 1.0.

Conversational-based interactivity. In this study, we used the operational definition of conversational interactivity to indicate McDonald's starting a brief conversation with a user on Facebook. McDonald's did not plot nor use computers to autogenerate these short conversations; they respond according to users' previous comments.

In the current study, conversational-based interactivity consists of two features: a) addressing users by name and b) providing direct answers to users' questions. Only when McDonald's replies were something like "Hi, Carlos. Thank you for your question. We don't add any hormones to our chicken. No one in the U.S. does...", do they count as conversational-based interactivity. Likewise, we assigned the number "1" to such conversational interactivity. We assigned the number " 0 " when they did not use such interactivity. The intercoder reliability is 1.0.

Process-based interactivity. The operational definition of process-based interactivity in this study is McDonald's not only starting a brief conversation with users but also providing them with hyperlinks to help users better understand the problem. We applied the same coding scheme. When they used process-based interactivity, we assigned the number " 1 ." When they did not, we assigned the number " 0 ." The intercoder reliability is 1.0 .

Participative social-interactive engagement. We measured participative socialinteractive engagement by the number of online comments followed by interactivity and by the number of "likes."Regarding the online commenting interactivity, the coders counted comments when, for example, they appeared after posted hyperlinks or if conversations started. The intercoder reliability is 1.0.The "like" number refers to the number of "likes" on McDonald's responses. For example, after McDonald's replies to a user's question, if other users click the "like" button under this response, the coders counted the number of likes. The intercoder reliability is 1.0.

Emotional social-interactive engagement. We measured emotional socialinteractivity by assessing the affective valence expressed in users' comments. We analyzed every sentence in each comment by extracting the core phrases in each sentence. A core phrase includes the subject, the predicate, and any objects of a sentence.

We set two standards to evaluate users' affective valences. First, the topic of the core phrase needed to relate to the food described in the theme of the campaign. For example, 
Online Journal of Communication and Media Technologies, 2019

Table 1. One-way ANOVA test results for online commenting numbers

\begin{tabular}{|c|c|c|c|c|c|}
\hline Variables & Interactivity & $N$ & $M$ & $S D$ & Sig \\
\hline \multirow[t]{6}{*}{ Online Commenting Numbers } & Medium-based interactivity & 119 & .62 & .66 & \multirow{2}{*}{.000} \\
\hline & Conversation-based interactivity & 124 & 1.38 & .72 & \\
\hline & Medium-based interactivity & 119 & .62 & .66 & \multirow{2}{*}{.000} \\
\hline & Process-based interactivity & 220 & 1.54 & .78 & \\
\hline & Conversation-based interactivity & 124 & 1.38 & .72 & \multirow{2}{*}{.187} \\
\hline & Process-based interactivity & 220 & 1.54 & .78 & \\
\hline
\end{tabular}

if the theme was people's concerns over the meat in McNuggets, then, we only coded the comments relating to McNuggets. Second, the predicate in the core phrase needed to describe the affective relationship between the McDonald's food or the company itself and its consumers. For example, if the sentence was "I love McNuggets," then we coded this sentence as positive. If the sentence was"I hate McNuggets," then, we coded this sentence as negative. If the sentence had both negative and positive feelings towards the food, then we coded the sentence as "neutral." The intercoder reliability is .81. We recorded the number of sentences of each affective valence, indicating the frequencies of each affective valence.

Statistical Analysis

We used the SPSS 22.0 to analyze the data. We employed ANOVA when the distribution of the data was parametric and normally distributed, and we used Tukey's HSD to determine the differences between the groups. When the distribution of the data was non-parametric, then we used Kruskal Wallis tests to analyze the data.

\section{RESULTS}

We collected a total of 1,000 comments from the Facebook platform. To fulfill the requirement of normal distribution, we used log transformation for the frequencies of the number of online commenting and the number of "likes." After the log transformation, the skew and kurtosis values of these continuous variables were within the range of -2 to 2 , satisfying the requirement for a reasonably normal distribution (Bachman, 2004). Based on the data sets, we conducted the following analyses.

To answer RQ1, we investigated the differences in the number of certain online comments and the number of "likes" among these three types of interactivity. A total of $233(23.3 \%)$ responses exhibited process-based interactivity, 140 (14\%) responses reflected conversational-based interactivity, and 156 (15.6\%) responses displayed medium-based interactivity.

To examine the differences in the numbers of online commenting among the three types of interactivity, we used a one-way ANOVA (See Table 1). The results show a significant difference among the groups $(F(3,593)=51.98, p<.001)$. We used Tukey's HSD to determine the differences between the groups. The analysis showed that conversationbased interactivity $(M=1.38, S D=.72)$ generated significantly greater online commenting numbers than did medium-based interactivity $(M=.62, S D=.66, p<.001)$. Process-based interactivity $(M=1.54, S D=.78)$ prompted significantly greater online commenting numbers than did medium-based interactivity $(M=.62, S D=.66, p<.001)$. We found no significant difference between process-based interactivity $(M=1.54, S D=$ $.78)$ and conversation-based interactivity $(M=1.38, S D=.72, p>.05)$ in terms of online commenting numbers.

Likewise, to examine the differences of the number of "likes" among the three types of interactivity, we used a one-way ANOVA (See Table 2). We found a significant difference 
F. Qiao

Table 2. One-way ANOVA test results for "like" numbers

\begin{tabular}{|c|c|c|c|c|c|}
\hline Variables & Interactivity & $N$ & $M$ & $S D$ & Sig \\
\hline \multirow[t]{6}{*}{ "like" numbers } & Medium-based interactivity & 73 & .41 & .58 & \multirow{2}{*}{.000} \\
\hline & Conversation-based interactivity & 76 & 1.0 & .90 & \\
\hline & Medium-based interactivity & 73 & .41 & .58 & \multirow{2}{*}{.000} \\
\hline & Process-based interactivity & 249 & 1.14 & .98 & \\
\hline & Conversation-based interactivity & 76 & 1.0 & .90 & \multirow{2}{*}{.603} \\
\hline & Process-based interactivity & 249 & 1.14 & .98 & \\
\hline
\end{tabular}

Table 3. Kruskal-Wallis Test Results

\begin{tabular}{|c|c|c|c|c|c|}
\hline Variable & Interactivity & $N$ & Mean Rank & Chi-Square & Sig \\
\hline \multirow[t]{6}{*}{ Positive Affective valences } & Medium-based interactivity & 118 & 104.31 & \multirow{2}{*}{23.35} & \multirow{2}{*}{.000} \\
\hline & Conversation-based interactivity & 120 & 134.43 & & \\
\hline & Medium-based interactivity & 118 & 168.61 & \multirow{2}{*}{49.98} & \multirow{2}{*}{.000} \\
\hline & Process-based interactivity & 344 & 253.07 & & \\
\hline & Conversation-based interactivity & 120 & 211.50 & \multirow{2}{*}{5.15} & \multirow{2}{*}{.023} \\
\hline & Process-based interactivity & 344 & 239.82 & & \\
\hline
\end{tabular}

among the groups $(F(3,474)=21.01, p<.001)$. We used Tukey's HSD to determine the differences between the groups. The results show that conversation-based interactivity $(M=1.0, S D=.90)$ generated a significantly greater number of "likes" than did mediumbased interactivity $(M=.41, S D=.58, p<.001)$. Process-based interactivity $(M=1.14, S D$ $=.98$ ) produced a significantly greater number of "likes" than did medium-based interactivity $(M=.41, S D=.58, p<.001)$. In terms of the number of "likes," we found no significant differences between process-based interactivity $(M=1.14, S D=.98)$ and conversation-based interactivity $(M=1.0, S D=.90, p>.05)$.

To answer RQ 2, we investigated how the different types of interactivity influenced consumers' engagement with the emotional-social interaction. The frequencies of each affective valence were not normally distributed. Also, the frequencies still failed to fulfill the requirement of reasonably normal distribution after the log transformation. Therefore, we used a series of non-parametric Kruskal-Wallis tests to explore this research question (See Table 3). In terms of negative affective valences, we found no significant difference among the three types of interactivity.

In terms of positive affective valences, we found a significant difference between conversation-based interactivity and medium-based interactivity $(H(1)=23.35, p<.001)$. The conversation-based interactivity had an average positive affective valence of 134.43 while the medium-based interactivity had an average positive affective valence of 104.31. In other words, conversation-based interactivity generated significantly more positive affective valences than did medium-based interactivity.

Also, we found a significant difference between medium-based interactivity and process-based interactivity $(H(1)=49.98, p<.001)$. Medium-based interactivity had an average positive affective valence of 168.61 while process-based interactivity had an average positive affective valence of 253.07. Process-based interactivity generated significantly more positive affective valences than did medium-based interactivity.

In addition, we found a significant difference between the conversation-based and the process-based interactivity strategies $(H(1)=5.154, p<.05)$. Conversation-based interactivity had an average positive affective valence of 211.50 while process-based interactivity had an average positive affective valence of 239.82. Process-based interactivity generated significantly more positive affective valences than did conversation-based interactivity. Based on the aforementioned results, process-based 
Online Journal of Communication and Media Technologies, 2019

interactivity prompted the greatest positive affective valences among the three types of interactivity.

\section{DISCUSSION}

Through conducting a case study on McDonald's recent released global campaign "Our Food, Your Questions," we set out to better understand different types of interactivity on social media, especially for those at the content-community level of social media, such as Facebook. We began this study by integrating previous interactivity research, by observing a real-world global campaign, and by conceptualizing interactivity on a continuum from low-order to high-order. Then, we proposed a distinction among mediumbased, conversation-based, and process-based types of interactivity. Furthermore, we conducted a quantitative research to examine the effects of these types of interactivity on consumers' engagement with social-interactivity.

The results offered empirical support to our conceptual framework for the different types of interactivity on higher-level social media platforms. To sum up, conversationbased interactivity generated greater participative and emotional social-engagement than did medium-based interactivity. Also, process-based interactivity produced more positive emotional social-engagement than did either the conversation-based or medium-based types of interactivity.

The results relate to the typology of consumers' needs for using social media (Kang, Tang, \& Fiore, 2014). Kang et al. (2014) proposed four benefits that consumers gain via online community interactions, including a) functional benefits, referring to consumers' needs to obtain information in an online community, b) social-psychological benefits, meaning consumers' expectations of social rewards through the interaction, c) hedonic benefits, referring to consumers' explorations for fun and enjoyment, and d) monetary benefits, meaning the consumers' experiences with the long-term services from the company. Based on the results of the current research, the medium-based interactivity mainly fulfilled consumers' functional benefits because hyperlinks provide consumers with direct and straightforward information about McDonald's food quality. Conversation-based interactivity satisfied the consumers' social-psychological benefits as they expected to receive help from the brand through the online interactions. The reciprocal communication with McDonald's not only enabled the brand to extend their assistance and support for consumers to ease their doubts about the food quality but also created a sense of belonging for consumers to feel identified as active participants in the campaign. The process-based interactivity and the combination of medium- and conversation-based interactivity satisfied both functional and social-psychological benefits. In addition, the process-based type of interactivity attempted to fulfill consumers' relational benefits - the core value of monetary benefits (Kang et al., 2014) because the consumers focused not only on the factual information about the food quality-the centerpiece of McDonald's services-but also on the satisfaction they received from conversations with the brand, the emotional attachment gained through obtaining social and respect-based rewards via interactions, resulting in a more cohesive consumer-brand relationship. Yet, one could consider the campaign we examined in the current study to be a response strategy to combat consumers' doubts about its food quality. Thus, conversation-based or even process-based interactivity cannot result in consumers' hedonic benefits because the employment of entertaining content, indicating fun or pleasure (Wang \& Fesenmaier, 2004), would be inappropriate and inconsistent with the 
theme of this transparent campaign. Therefore, researchers could further investigate the benefits gained by these three types of interactivity by comparing different campaigns.

Building on the conceptualization of interactivity in the context of the contentcommunity level of social media, we established that scholars can more accurately distinguish different types and levels of interactivity. In this study, we presented evidence that social-interactive engagement manifest itself differently through different types of interactivity. In the current study, we mainly focused on the medium of hyperlinks, and we would encourage future scholars to continue to explore the complexities of interactivity on various social media platforms. In an example of medium-based interactivity, sharing photos could prompt different social engagement effects than would sharing hyperlinks because people might adopt different mechanisms to process visual- and text-based information (Hasson, Chen, \& Honey, 2015).

Also, differences might exist in the emotional-social engagement effects if different combination orders occurred in process-based interactivity. For example, consumers might feel more satisfied if conversation-based interactivity appeared prior to mediumbased interactivity, as compared to the other way around, since our results show that conversation-based interactivity has a greater capacity to generate more positive emotion than does medium-based interactivity. Conducting future empirical analyses to compare the order effects of process-based interactivity as well would be interesting.

From the applied perspective, businesses are encouraged to use process-based and conversation-based interactivity. Businesses seeking to maintain relations with their customers can look at the evidence in the current study to see that users have greater participative and emotional social-interactive engagement if they, the businesses, use these two types of interactivity. Having a better electronic word of mouth (eWOM) was also positive in a normative sense, where positive eWOM might indicate more satisfied consumers, as well as a greater purchase intention.

\section{IMPLICATIONS AND CONCLUSIONS}

The current study revealed several practical implications for businesses' online community management. First, medium-based interactivity might be a more time-saving relational maintenance strategy for businesses, compared to the other two types of interactivity, because it would take less effort to share a hyperlink or a picture than to start a conversation. Also, it might also better help users to locate the appropriate information in a straightforward manner without any socializing greetings. Second, conversation-based interactivity might be more time-consuming for companies to maintain their relations with consumers; however, stronger brand-consumer connections might emerge, resulting in improving consumers' lifetime value with the brand and prompting active community participation. Yet, the beneficial effects of conversationbased interactivity appeared only when genuine conversations occurred. Genuine conversation needs to be based on a) the positive input, experiences, and needs both of the brands and of the consumers, b) communication for relational purposes, and c) consumers' advice on issues of community concern (Taylor \& Kent, 2014).

For example, if the companies initiated conversations and only followed the same pattern, using exactly the same sentences or words without any personalization or adaptation, to respond to every consumer's questions, the consumers might not perceive such conversations as genuine because such conversations do not fill any of the consumers' needs, the company has done little towards sustaining relational maintenance, and they are showing no effort to take their consumers' advice. In such a situation, consumers are 
Online Journal of Communication and Media Technologies, 2019

\begin{tabular}{|c|c|c|c|}
\hline Strategy & Expected Results & Tradeoffs & Exceptions \\
\hline $\begin{array}{l}\text { Medium- } \\
\text { based } \\
\text { interactivity }\end{array}$ & $\begin{array}{l}\text { a) The least online commenting } \\
\text { numbers } \\
\text { b) The least "like" numbers } \\
\text { c) The least positive response from } \\
\text { consumers }\end{array}$ & $\begin{array}{l}\text { a) Time-saving } \\
\text { b) Direct delivery of factual } \\
\text { information } \\
\text { c) Fulfillment of consumers' } \\
\text { functional needs }\end{array}$ & $\begin{array}{l}\text { Photo sharing might } \\
\text { increase its effects }\end{array}$ \\
\hline $\begin{array}{l}\text { Conversation- } \\
\text { based } \\
\text { interactivity }\end{array}$ & $\begin{array}{l}\text { a) The second best online commenting } \\
\text { numbers } \\
\text { b) The second best "like" numbers } \\
\text { c) The second best positive response } \\
\text { from consumers }\end{array}$ & $\begin{array}{l}\text { a) Time-consuming } \\
\text { b) Establishment of stronger } \\
\text { brand-consumer relationship } \\
\text { c) Fulfillment of consumers' } \\
\text { social-psychological needs }\end{array}$ & $\begin{array}{l}\text { "Non-genuine" or } \\
\text { "impersonalized" } \\
\text { conversation might } \\
\text { decrease its effects }\end{array}$ \\
\hline $\begin{array}{l}\text { Process-based } \\
\text { interactivity }\end{array}$ & $\begin{array}{l}\text { a) The second best online commenting } \\
\text { numbers (no statistical difference from } \\
\text { conversation-based interactivity) } \\
\text { b) The second best "like" numbers (no } \\
\text { statistical difference from conversation- } \\
\text { based interactivity) } \\
\text { c) The best positive response from } \\
\text { consumers }\end{array}$ & $\begin{array}{l}\text { a) Time-consuming } \\
\text { b) Establishment of stronger } \\
\text { brand-consumer relationship } \\
\text { c) Fulfillment of consumers' } \\
\text { relational/monetary needs }\end{array}$ & $\begin{array}{l}\text { The order of the } \\
\text { process might } \\
\text { intervene its effects }\end{array}$ \\
\hline
\end{tabular}

likely to ridicule conversation-based interactivity, which results in decreasing the benefits of the two-way interactions. In this sense, medium-based interactivity serves a better function than does the non-genuine conversation-based interactivity.

Table 4 shows a summary of the benefits, tradeoffs, and exceptions of each type of interactivity. The employment of a particular type of interactivity might depend on the situation. It would be interesting for scholars to further explore which type of interactivity could better fit different situation types.

\section{Limitations}

For this study, we took an empirical approach to examine the effects of three types of conceptualized interactivity on users' engagement with online social interactions. Future research could also benefit from addressing several limitations of the current study. First, we only analyzed one recent global campaign. Future research could include more different types of campaigns, such as campaigns in response to crisis situations, to further examine the relationship between campaign types and interactivity. Second, we only conducted an analysis on one type of content-social community. Expanding the research to compare the effects of interactivity across different content social communities, like Instagram, Snapchat, Twitter, etc. would be useful. Investigations like this could further validate or falsify the conceptualization we've proposed in this research, pushing forward our advancement of knowledge in this field. Finally, while it might be common to quantify interactivity and social-interactive engagement, future qualitative research on why businesses use different relational maintenance strategies and how consumers perceive those strategies would greatly expand the scope of the current study. Conceptualizing interactivity on the content-community level of social media contributed to a richer understanding of the extant research in the realm of social media, and the effects we investigated in the current study would offer guidance on interactivity for relational maintenance practitioners. But even as the current study helped advance our knowledge of interactivity on social media, many questions still remain unanswered and need to be explored in the future. 


\section{ACKNOWLEDGEMENT}

This study is supported by the research project of 2017 Guangzhou Center for Innovative Communication in International Cities in Guangzhou's 13th Five-Year Plan for the Development of Philosophy and Social Science under Grant No. 2017-JD-01.

\section{REFERENCES}

Aguilhar, L. (2018). 15 social media statistics you need to know. Strike Social. Retrieved from https://strikesocial.com/blog/15-social-media-marketing-statistics-you-need-toknow/

Aikat, D. (2000). A new medium for organizational communication: Analyzing web content characteristics of Fortune 500 companies. Electronic Journal of Communication, 10(1), 2.

Bachman, L. F. (2004). Statistical analyses for language assessment. Edinburgh: Cambridge University Press. https://doi.org/10.1017/CBO9780511667350

Daft, R. L., \& Lengel, R. H. (1986). Organizational information requirements, media richness and structural design. Management Science, 32(5), 554-571. https://doi.org/10.1287/mnsc.32.5.554

Facebook. (2017). What does it mean to "like" something? Retrieved from https://www.facebook.com/help/110920455663362

Gerlitz, C., \& Helmond, A. (2013). The like economy: Social buttons and the data-intensive web. New media \& society, 15(8), 1348-1365. https://doi.org/10.1177/1461444812472322

Hainla, L. (2018). 21 Social media marketing statistics you need to know in 2018. Dream Grow. Retrieved from https://www.dreamgrow.com/21-social-media-marketingstatistics/

Hasson, U., Chen, J., \& Honey, C. J. (2015). Hierarchical process memory: Memory as an integral component of information processing. Trends in Cognitive Sciences, 19(6), 304-313. https://doi.org/10.1016/j.tics.2015.04.006

Kang, J., Tang, L., \& Fiore, A. M. (2014). Enhancing consumer-brand relationships on restaurant Facebook fan pages: Maximizing consumer benefits and increasing active participation. International Journal of Hospitality Management, 36, 145-155. https://doi.org/10.1016/j.ijhm.2013.08.015

Kaplan, A. M., \& Haenlein, M. (2010). Users of the world, unite! The challenges and opportunities of social media. Business Horizons, 53(1), 59-68. https://doi.org/10.1016/j.ijhm.2013.08.015

Kelleher, T. (2009). Conversational voice, communicated commitment, and public relations outcomes in interactive online communication. Journal of Communication, 59(1), 172-188. https://doi.org/10.1111/j.1460-2466.2008.01410.x

Ksiazek, T. B., Peer, L., \& Lessard, K. (2016). User engagement with online news: Conceptualizing interactivity and exploring the relationship between online news videos and user comments. New Media \& Society, 18(3), 502-520. https://doi.org/10.1177/1461444814545073

Liu, Y., \& Shrum, L. (2002). What is interactivity and is it always such a good thing? Implications of definition, person, and situation for the influence of interactivity on advertising effectiveness. Journal of Advertising, 31(4), 53-64. https://doi.org/10.1080/00913367.2002.10673685 
Online Journal of Communication and Media Technologies, 2019

McMillan, S.J. (2002) 'Exploring Models of Interactivity from Multiple ResearchTraditions: Users, Documents, and Systems', in L. Lievrouw and S. Livingstone (eds). The Handbook of New Media: Social Shaping and Consequences of ICTs, pp. 163-82. London: Sage. https://doi.org/10.4135/9781446206904.n13

Mersey, R. D., Malthouse, E. C., \& Calder, B. J. (2010). Engagement with online media. Journal of Media Business https://doi.org/10.1080/16522354.2010.11073506

Rafaeli, S., \& Sudweeks, F. (1997) Networked Interactivity, Journal of ComputerMediated Communication, 2(4), https://doi.org/10.1111/j.1083-6101.1997.tb00201.x

Salomon, G. (1997) Of mind and media, Phi Delta Kappa, 78(5), 375-81.

Schultz, F., Utz, S., \& Göritz, A. (2011). Is the medium the message? Perceptions of and reactions to crisis communication via twitter, blogs and traditional media. Public Relations Review, 37(1), 20-27. https://doi.org/10.1016/j.pubrev.2010.12.001

Short, J., Williams, E., \& Christie, B. (1976). The social psychology of telecommunications. London; New York: Wiley.

Starkman, N. (2014). What McDonald's new “transparency”campaign is hiding. Retrieved from http://time.com/3501921/mcdonalds-transparency-campaign/

Sundar, S. S., Kalyanaraman, S., \& Brown, J. (2003). Explicating web site interactivity impression formation effects in political campaign sites. Communication Research, 30(1), 30-59. https://doi.org/10.1177/0093650202239025

Taylor, M., \& Kent, M. L. (2014). Dialogic engagement: Clarifying foundational concepts. Journal of Public Relations Research, 26(5), 384-398. https://doi.org/10.1080/1062726X.2014.956106

TechTerms.com. (2014). Hyperlinks. Retrieved from http://techterms.com/definition/hyperlink

Tremayne, M. (2008). Manipulating interactivity with thematically hyperlinked news texts: Amedia learning experiment. New Media \& Society, 10(5), 703-727. https://doi.org/10.1177/1461444808094353

Walther, J. B. (1994) Anticipated Ongoing Interaction versus Channel Effects on Relational Communication in Computer - Mediated Interaction, Human Communication Research 20,473-501. https://doi.org/10.1111/j.14682958.1994.tb00332.x

Wang, Y., \& Fesenmaier, D. R. (2004). Towards understanding members' general participation in and active contribution to an online travel community. Tourism Management, 25(6), 709-722. https://doi.org/10.1016/j.tourman.2003.09.011

Wang, Y., Qiao, F., \& Peng, W. (2015). Is the size or the valence of proactive engagement associated with purchase intention? A case study of branded blogs of Starbucks. International Journal of Strategic Communication, 9(3), 197-216. https://doi.org/10.1080/1553118X.2014.924125

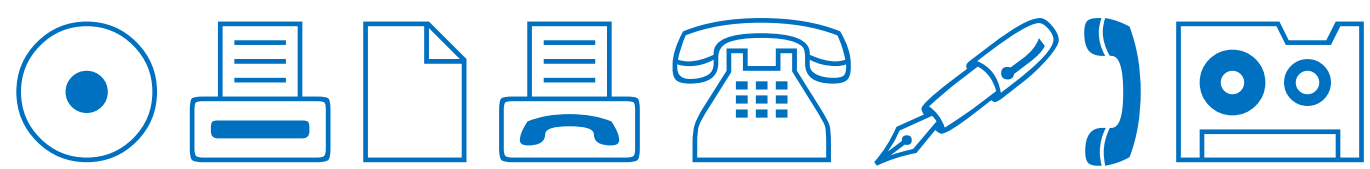

